\title{
Application of the bi-rads classification for ultrasound diagnosis of breast diseases
}

\author{
Ismailova Munojat Khayatovna \\ Tashkent medical academy \\ Email address: \\ munojat1977@mail.ru (Ismailova Munojat Khayatovna)
}

To cite this article:

Ismailova Munojat Khayatovna. Application of the bi-rads classification for ultrasound diagnosis of breast diseases. Journal of research in health science. Vol. 2, No. 2, 2017, pp. 15-21. DOI $10.26739 / 2523-1243$

\section{dol http://dx.doi.org/10.26739/2523-1243/-2017-2-2-4}

\begin{abstract}
: currently, a mayor problem in the clinical diagnosis of breast care is fibrocystic breast to mammologist such patients were treated normally after the ultrasound examination with a diagnosis of "fibrocystic breast disease". With a view to strengthening the role of ultrasound diagnostics authors for practitioners offer ultrasound algorithm for patients with disease of the breast using a classification by BI-RADS, which allows you to accurately determine the changes in the breast tissue.
\end{abstract}

Keywords: fibrous breast, ultrasound, local fibrosis, cyst, breast cancer.

\section{Применение классификации BI-RADS при ультразвуковой диагностике заболеваний молочных желез}

Анотация: в настоящее время в маммологии большую проблему представляет диагностика фиброзно-кистозной мастопатии, к маммологу такие пациенты обращаются обычно после ультразвукового исследования с диагнозом " фиброзно- кистозная мастопатия; мастопатия, узловая форма".система BI-RADS позволяет с высокой точностью определить характер заболеваний молочной железы и выбрать оптимальную тактику лечения.

Ключевое слова: фиброзная мастопатия, ультразвуковая диагностика, локальный фиброз, киста, рак молочной железы. 
Ismailova Munojat Khayatovna. Application of the bi-rads classification for ultrasound diagnosis of breast diseases.

\section{АКТУАЛЬНОСТЬ ПРОБЛЕМЫ.}

На практике врач маммолог сталкивается с диагнозом фибрознокистозной мастопатии после ультразвукового исследования. В то время как, на основании результата рентгеновской маммографии дается несколько другое заключение. В данной работе предлагается оценивать заключение ультразвукового исследования и рентгеновской маммографии по единой системе.

С целью усиления роли Уз диагностики авторы для практических врачей предлагают алгоритм УЗИ для пациентов с заболеваниями молочных желез с применением классификацию по BI-RADS, который позволяет с высокой точностью определить изменений в ткани молочной железы.

Для уточнения структуры очагового - фиброзного узла требуется пункционная биопсия, которая позволяет верифицировать морфологическую структуру узла и определить характер образования. (6)

По данным авторов, точность ультразвуковой диагностики узловой формы мастопатии при использовании датчика для исследования 7,5 МГц составляет 80-85\%.(7)

Истинная природа узлового компонента определяется комплексом современных лучевых и патоморфологических методов исследовании. Можно выделить широкий круг заболеваний , скрывающихся под маской узловой мастопатии: локальный фиброз - 45\%, киста - 22\%, фиброаденома - 17\%, липома -10\%, РМЖ, внутрипротоковая папиллома, интраммарный лимфатический узел т.д.(8)
В настоящее время в клинической маммологии большую проблему представляет диагностика фибрознокистозной мастопатии, к маммологу такие пациенты обращаются обычно после ультразвукового исследования с диагнозом " фиброзно- кистозная мастопатия; мастопатия, узловая форма".

В настоящее время среди клиницистов сложилось единое мнение, что диагноз " узловая форма" мастопатии правомочен лишь на первоначальном этапе обследования и должен получить конкретное определение патологии после комплексного обследования, включающего помимо УЗИ и РМ. При необходимости аспирационную пункционную биопсию. Это позволяет в большинстве случаев достаточно аргументировано исключить или подвердить наличие в молочной железе злокачественной опухоли.

В клинической практике основным методом объективной оценки состояния молочных желез и диагностики патологии является РМ, которая обеспечивает документацию изменений в молочной железе и динамическое наблюдение за ее состоянием $(3,4,5)$

В последние десятилетия успешно работают скрининговые маммографические программы, позволяющие выявлять минимальные изменения в виде образований в молочной железе, подозрительные в отношения злокачественного роста.(1)

По данным литературы, с помощью РМ можно своевременно распознат»ь патологические изменения и образования в молочных железах в 85-97 \% случаев, а 
достоверность маммографического исследования в диагностике рака молочной железы колеблется в диапазоне от 75 до $95 \%(2,4)$.

Однако в клинической маммологии и в практике врача УЗИ отмечается недостаточно рекомендаций и конкретных алгоритмов обследования пациентов с очаговыми образованиями молочных желез с применением системы BI-RADS.

\section{ЦЕЛЬ ИССЛЕДОВАНИЯ}

Оценка молочных желез на основании УЗИ с применением классификации BI-RADS.

Материал и методы исследования. Проведенное нами исследование включало обследование 70 женщин с заболеваниями молочной железы, в течение периода с2016 по 2017 гг.

Возраст женщин от 30 до 70 лет. Средний возраст - 42 ё 0,2 лет.

Всем пациенткам было поведено рентгенологическое и ультразвуковое исследование.

Ультразвуковое исследование проводили на 7-12 -й день менструального цикла (при его регулярности ) на аппарате MINDRAY - DC 3 в реальном масштабе времени линейным датчиком с частотой 7,5 МГц.

Рентгенологическое исследование молочных желез выполняли на аппарате Fujifilm Amulet S , в двух стандартных проекциях.

Поводом для обращения послужил дискомфорт, нагрубание, диффузное или локальное уплотнение, боль в молочной железы, выделение из соска.

Характер болевого симптома был различным. У 16 (22,9\%) женщин- они были постоянными, у $12(17,4 \%)$ - имели передменструальный характер, у $8(11,4 \%)$ возникли в период менструации. у $5(7,1 \%)$ - боль была наиболее выражена и боль иррадирует в плечо и лопатку. Выделение из соков было у 2 (2,9\%) женщин.

В репродуктивным возрасте находились пациентки 42 (60\%), в менопаузе - 28 (40\%). У 40 (54,8\%) женщины менструальный цикл был регулярным. Менструирующие женщины обследованы на 7-12 день менструального цикла.

Из 70 женщин у 35 (50\%) отмечалось лимфоаденопатия.

Из 70 женщин у $55 \quad(78,6 \%)$ отмечались микрокальцинатов. Из 55 них $10(18,2 \%)$ было гетерогенных и полеморфных микрокальцинатов.

У $14(21,4 \%)$ с выявленными очаговыми образованиями была выполнена тонкоигольная аспирационная биопсия.

Исследование было проведено на базе Республиканского научного центра онкология МЗ РУз.

Результаты и обсуждение.

Пациенты , в соответствие с проведенным РM очаговых образований и морфологическим заключениям, распределились следующим образом.

BI-RADS 1 - отсутствие очаговых изменений ( рутинное ежегодное обследование ) были выявлены у 10 $(14,4 \%)$

BI-RADS 2- доброкачественные изменения (кисты, липомы, интармаммарные лимфатические узлы, типичные фиброаденомы безусиления линейного размера за период наблюдения неменее 6 месяцев) - были выявлены у 29 $(41,4 \%)$ 
Ismailova Munojat Khayatovna. Application of the bi-rads classification for ultrasound diagnosis of breast diseases.

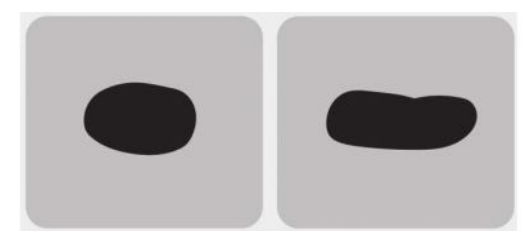

Схематическое изображение

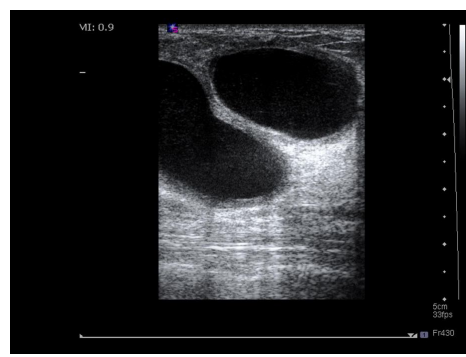

Пациентка М., 35 лет. УЗИ заключение:

фиброзно- кистозная мастопатия

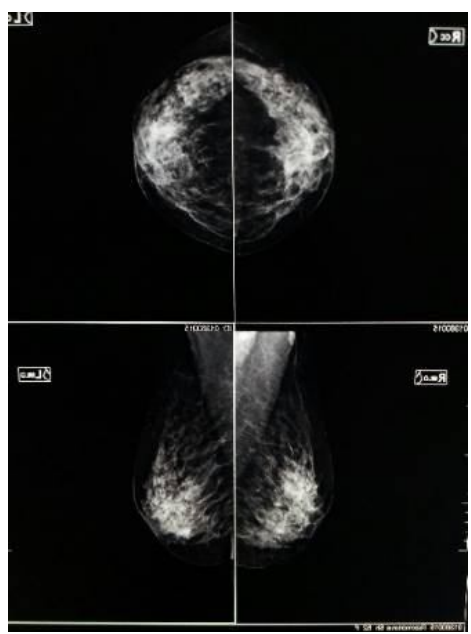

Маммография,той же пациентки по РМ заключение :

$$
\text { BI-RADS } 2
$$

BI-RADS- 3, доброкачественные изменения подозрением на рак не более $2 \%$ ( впервые с выявленные фиброаденомы , зоны узловой гиперплазии паренхимы)

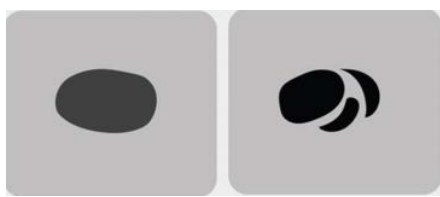

Схематическое изображение

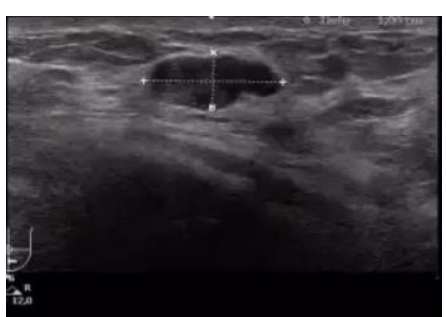

Пациентка М., 28 лет. УЗИ заключение :

фиброаденома правой молочной железы.

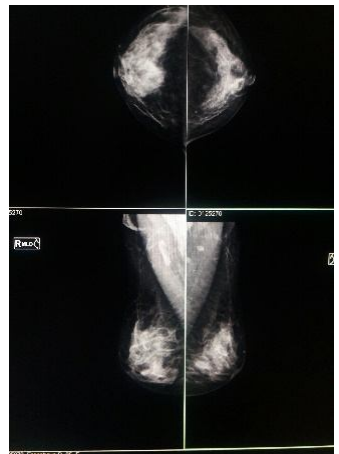

Маммография в двух проекциях, той же пациентки по

PM заключение: BI-RADS 3 правой молочной железы.

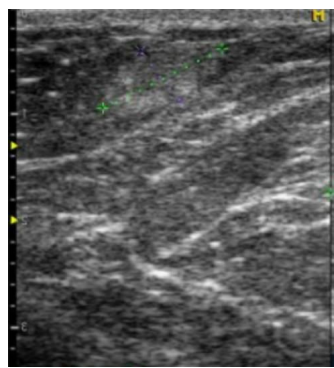

Пациентка Н., 37 лет . УЗИ заключение: мастопатия, узловая форма правой молочной железы. 


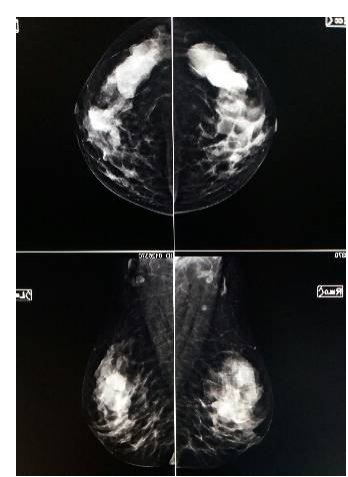

\section{Маммография той же пациентки} РМ заключение:

BI-RADS 3 правой молочной железы

BI-RADS- 4., 4a 4b 4c - выявленные изменения подозрительны на злокачественный процесс с вероятностью от $2 \%$ до 94\%; разделяют низкую, среднюю и высокую степень вероятности рака. (Категории 4a 4b 4c соответственно). (Морфологическая верификация диагноза путем выполнения чрескожной пункционной биопсии). Были выявлены у $8(11,3 \%)$
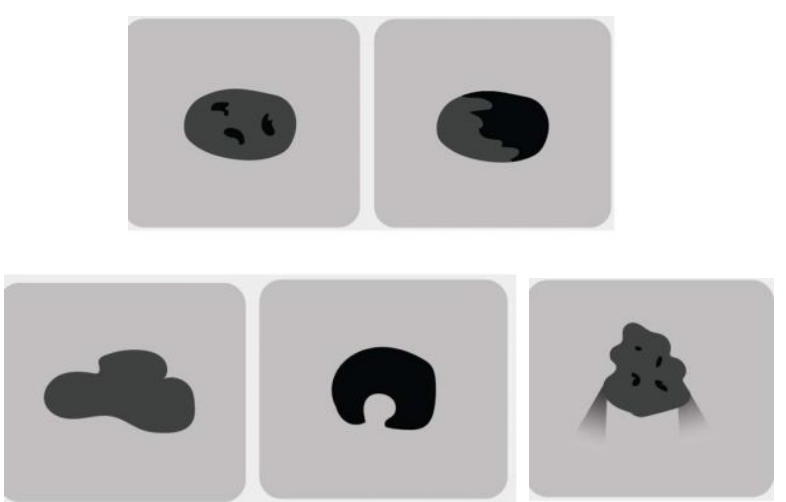

Схематически изображений для категории 4.

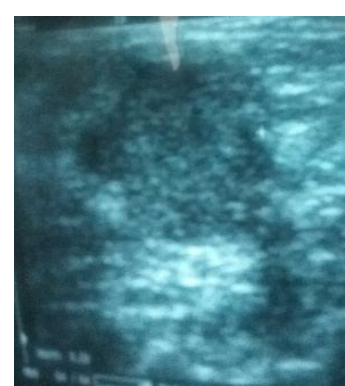

Пациентка М., 46 лет. УЗИ заключение: Susp. tumor левой молочной железы.

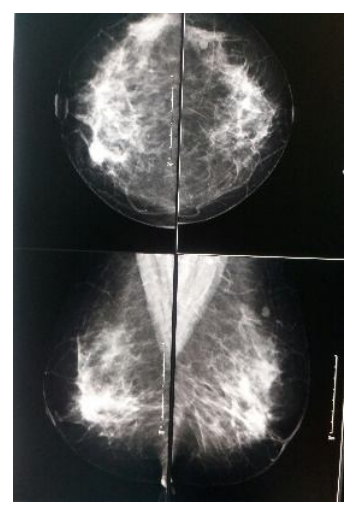

Маммография в двух проекциях.

РМ заключение:

\section{BI-RADS 4.}

После биопсии: атипичные клетки 3 степени.

В другом случае:

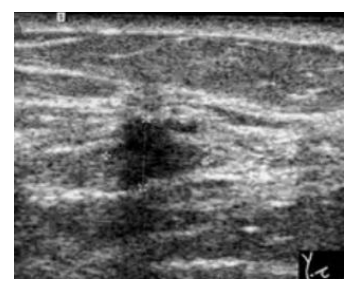

Пациентка Ш., 54 лет. УЗИ заключение: tumor левой молочной железы. 
Ismailova Munojat Khayatovna. Application of the bi-rads classification for ultrasound diagnosis of breast diseases.

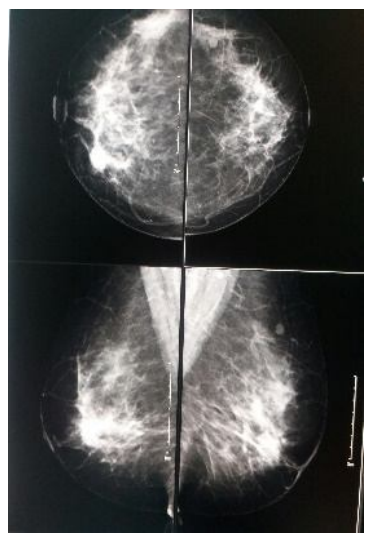

Маммография в двух проекциях. РМ заключение той же пациентки:

BI-RADS 4b. Морфологически: протоковый канцер.

BI-RADS -5 явные признаки злокачественных изменений с вероятностью $95 \%$ и выше. Морфологическая верификация диагноза выполнена путем чрескожной пункционной биопсии. Были выявлены у 7 (10\%) женщин.

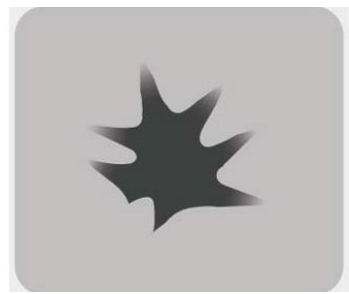

\section{Схематически изображение для категории 5}

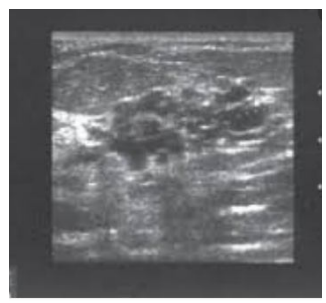

Пациентка У., 56 лет. УЗИ заключение: образование правой молочной железы, размерами 24х29 мм, с неровными контурами.

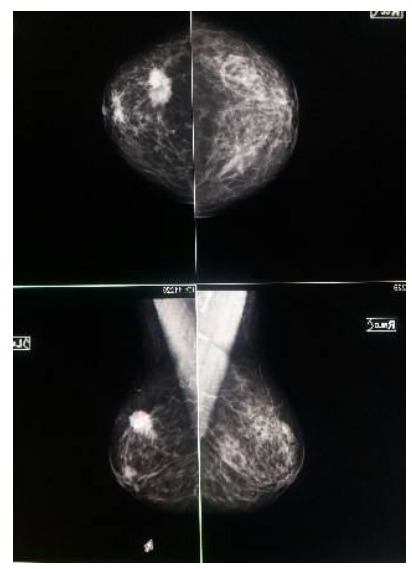

PМ той же пациентки: в верхненаружном квадранте определяется образование, размерами $27 \times 31$ мм, с лучистыми контурами. Заключение:

BI-RADS -5 правой молочной железы. После биопсии: инфильтрирующий канщер.

BI-RADS -6 - диагноз рака доказан морфологически. Устанавливается перед началом проведения специального лечения, включающего неадъювантную химиотерапию и хирургическое лечение.

Были выявлены у 4(5,6\%).

Таким образом, система BI-RADS позволяет с высокой точностью определить характер заболеваний молочной железы и выбрать оптимальную тактику лечения.

\section{ВЫВОДЫ:}

1. Диагностика заболеваний молочной железы должно быть комплексной, состоящей из клинического обследования, рентгеновской маммографии, ультразвукового и патоморфологического исследований.

2. Предложенные критерии УЗИ помощью BI-RADS помогают с высокой точности определить изменений в ткани молочной железы. 


\section{ЛИТЕРАТУРА:}

1. Васильев Д. А., Зайцев А.Н., Берштейн Л.М., "Маммографическая плотность молочных желез и определяющий ее факторы в свете повышенного онкологического риска"// "Опухоли женской репродуктивной системы". 2011.№3.С.33-36.

2. Гусейнов А.З., Истомин Д.А., "Заболевания молочной железы". Тула: изд-во ТулГУ , 2011. 211-234 стр.

3. Корженкова Г.П. Комплексная рентгено-сонографическая диагностика заболеваний молочной железы. (Учебно-методическое пособие). 2004. Под ред. Н.В.Кочергеной.

4. Летягин В.П., Высоцкая И.В., Ким Е.А. Факторы риска развития рака молочной железы. // Маммология. 2006. №4. 10-12 стр.

5. Одинцов В.А., Одинцова А.З., Гусейнов А.З., " Алгоритм диагностики не пальпируемых очаговых образований молочной железы с применением компьютерной программы моделирования" Вестник новых медицинских технологий. 2015.№3.

6. Рожкова Н. И., Кочетова Г. П. Особенности работы маммографического кабинета с CRкомплексом для цифровой маммографии // Мед.техн. 2007. № 5. 32-35 стр. 